\title{
THE AUTOMORPHISM GROUPS OF ALMOST HERMITIAN MANIFOLDS
}

\author{
BY \\ SHÛKICHI TANNO
}

1. Introduction. The maximum dimension of the group of isometries of an $m$-dimensional connected Riemannian manifold is $m(m+1) / 2$. And if the maximum is attained, then the Riemannian manifold is of constant curvature, and is one of the standard spaces: an $m$-dimensional sphere $S^{m}$, an $m$-dimensional real projective space $P^{m}$, an $m$-dimensional Euclidean space $E^{m}$, and an $m$-dimensional simply connected hyperbolic space $H^{m}$ (cf. S. Kobayashi and K. Nomizu [11, p. 308]). The purpose of this paper is to consider similar problems in almost Hermitian manifolds.

We denote by $A(M)$ the automorphism group of a connected almost Hermitian manifold $M$ with an almost complex structure $J$ and an almost Hermitian metric $G$. If the dimension of $M$ is $2 n$, then the maximum dimension of $A(M)$ is $n(n+2)$, as is seen in Lemma 2.1. Under the assumption that $M$ is a Kaehlerian manifold, K. Yano and I. Mogi showed in [17] that $M$ admits local holomorphic free mobility if and only if it is of constant holomorphic sectional curvature. Noticing that the maximum dimensionality of $A(M)$ and axiom of holomorphic free mobility are equivalent, we generalize their result in Theorem 3.3, from which we have

TheORem A. A 2n-dimensional connected almost Hermitian manifold $M$ admits the automorphisin group $A(M)$ of the maximum dimension $n(n+2)$, if and only if $M$ is one of the following three spaces:

(i) A complex projective space $C P^{n}$ with a Fubini-Study metric.

(ii) A unitary space $C E^{n}$.

(iii) An open ball with a Kaehlerian structure of negative constant holomorphic sectional curvature.

Next if we remove an almost Hermitian metric, then the situations are very different. In general, the automorphism group of an almost complex manifold $M$, that is, the set $J(M)$ of all $J$-preserving transformations, is infinite dimensional. Under the assumption of compactness, it is a finite dimensional Lie group (W. M. Boothby, S. Kobayashi and H. C. Wang [2]). And it has been conjectured by H. Chu and S. Kobayashi in [4] that the maximum dimension of $J(M)$ of a compact $2 n$ dimensional almost complex manifold $M$ is $2 n(n+2)$, and attained only when $M$ is a complex projective space. Under the additional conditions, we show that the conjecture is true.

Received by the editors December 14, 1967 and, in revised form, February 26, 1968. 
THEOREM B. Let $M$ be either (i) a compact Kaehlerian manifold with the constant scalar curvature, or (ii) a compact Einstein almost Kaehlerian manifold with the constant scalar curvature. Then the maximum dimension of $J(M)$ is $2 n(n+2)$, and the maximum is attained if and only if $M$ is a complex projective space.

It may be worth noting that $2 n(n+2)$ is twice the maximum dimension of $A(M)$.

The author appreciates the referee's valuable criticism, and simplification of proofs, especially, of Lemma 3.1.

2. Preliminaries. An almost complex manifold $M$ is defined by a structure tensor $J$ of type $(1,1)$, satisfying $J J X=-X$ for any vector field $X$ on $M . M$ is almost Hermitian if, moreover, it has a Riemannian metric $G$ such that

$$
G(J X, J Y)=G(X, Y)
$$

holds for any vector fields $X$ and $Y$ on $M$. Then we have a 2 -form $W$ called the fundamental 2-form; $W(X, Y)=G(X, J Y)$. When the exterior derivative $d W$ of $W$ vanishes, $M$ is called an almost Kaehlerian manifold. Further, if $D J=0$ for the Riemannian connection $D$ defined by $G$, then $M$ is called a Kaehlerian manifold.

Now, let $M$ be a $2 n$-dimensional almost Hermitian manifold. Then the necessary and sufficient conditions for $X$ to be an infinitesimal automorphism are

$$
\begin{aligned}
\left(L_{X} G\right)_{j k} & =G_{k r} D_{j} X^{r}+G_{j r} D_{k} X^{r}=0, \\
\left(L_{X} J\right)_{j}^{i} & =X^{r} D_{r} J_{j}^{i}-J_{j}^{r} D_{r} X^{i}+J_{r}^{i} D_{j} X^{r}=0,
\end{aligned}
$$

where $L_{X}$ denotes the Lie derivation by $X$ and indices $i, j, k$, and $r$ run from 1 to $2 n$.

Take an arbitrary point $P$ of $M$ and first consider $X$ such that $X_{P}=0$. Then (2.2) and (2.3) show that $\left(0,\left(D_{j} X^{i}\right)_{P}\right)$ leaves $G_{P}$ and $J_{P}$ invariant, and hence the set of all $\left(0,\left(D_{j} X^{i}\right)_{P}\right)$ is contained in the Lie algebra of the unitary group $U(n)$. Thus the maximum dimension for this set is $n^{2}$. Next we consider the vector $X$ such that $X_{P} \neq 0$. Then the set of all such vectors is at most $2 n$-dimensional. Since any Killing vector field $X$ is completely determined by the values $\left(X^{i}\right)_{P}$ and $\left(D_{j} X^{i}\right)_{P}$ at a point $P$, we have

Lemma 2.1. Let $M$ be a 2n-dimensional almost Hermitian manifold. Then the maximum dimension of $A(M)$ is $n(n+2)$.

\section{The automorphism group of the maximum dimension.}

LEMMA 3.1. Let $M$ be a $2 n$-dimensional almost Hermitian manifold which admits the automorphism group $A(M)$ of the maximum dimension $n(n+2)$. If a tensor field $K$ of type $(r, s)$ is invariant by $A(M)$, then $K=0$ provided that $r+s$ is odd.

Proof. The linear isotropy group at $P$ of $A(M)$ may be considered as the unitary group $U(n)$, since $A(M)$ is of the maximum dimension. So it contains a 1-parameter group $e^{i t} I$, and, in particular, the element $-I$, where $I$ is an identity operator. And $-I$ maps $K$ into $(-I)^{r+s} K$. This implies that $K$ vanishes if $r+s$ is odd. 
Lemma 3.2. Assume that $A(M)$ of an almost Hermitian manifold $M$ is of the maximum dimension. Then $M$ is Kaehlerian.

Proof. Let $X$ be any infinitesimal automorphism on $M$. Since the Lie derivation by a Killing vector field commutes with the covariant differentiation, we have

$$
L_{X} D J=D L_{X} J=0 .
$$

Since $D J$ is a tensor field of type $(1,2)$ and invariant by any infinitesimal automorphism $X, D J$ vanishes by Lemma 3.1. Therefore $M$ is Kaehlerian. Q.E.D.

Take a point $P$ in an almost Hermitian manifold $M$. If the sectional curvature for the 2-plane defined by a tangent vector $Y_{P}$ at $P$ and $J Y_{P}$ is constant $k(P)$ for each $Y_{P}$, then $M$ is said to have constant holomorphic sectional curvature at $P$. When $k$ is constant on $M, M$ is called a space of constant holomorphic sectional curvature, or of constant unitary curvature (cf. [1], [17]). If a Kaehlerian manifold $M(2 n \geqq 4)$ has constant holomorphic sectional curvature $k(P)$ at each point $P$, then $k$ is constant on $M$, and equivalently the Riemannian curvature tensor is of the form (K. Yano and I. Mogi [17]):

$$
R_{h i j k}=(k / 4)\left[\left(G_{h k} G_{i j}-G_{h j} G_{i k}\right)+\left(J_{h k} J_{i j}-J_{h j} J_{i k}\right)-2 J_{h i} J_{j k}\right] .
$$

In the proof of the next theorem we need the following fact: If $k$ is equal to zero, then $M$ has zero Riemannian curvature, and if $k$ is nonzero, then Riemannian curvature of $M$ takes its value between $k$ and $k / 4$ as is derived from (3.1).

When the dimension of $M$ is $2, M$ is of constant holomorphic sectional curvature if and only if it is of constant curvature.

Next take a point $P$ of an almost Hermitian manifold $M$ and a tangent vector $Y_{P}$ at $P$. Suppose that, for any point $Q$ and any tangent vector $Z_{Q}$ at $Q$, there exists an isometry of $M$ (resp. of a neighborhood of $P$ to that of $Q$ ) which maps $P$ to $Q$, $Y_{P}$ to $Z_{Q}$ and $J Y_{P}$ to $J Z_{Q}$. Then $M$ is said to admit (resp. local) holomorphic free mobility. It is well known (K. Yano and I. Mogi [17]) that a Kaehlerian manifold has constant holomorphic sectional curvature if and only if it admits local holomorphic free mobility (where "local" is necessary, because they consider the solutions of the differential equations, but the extension of such solutions in local coordinate neighborhoods to the whole manifold is not always possible). The necessary and sufficient condition for an almost Hermitian manifold to admit holomorphic free mobility is given by the next theorem.

TheOREM 3.3. A 2n-dimensional almost Hermitian manifold $M$ admits the automorphism group of the maximum dimension $n(n+2)$, if and only if $M$ is a simply. connected, complete Kaehlerian manifold with constant holomorphic sectional curvature.

Proof. Assume that the dimension of $A(M)$ is $n(n+2)$. Then $M$ is Kaehlerian and, as (2.2) and (2.3) imply, $M$ is homogeneous and admits holomorphic free m sbility. Therefore $M$ has constant holomorphic sectional curvature $k$. First we 
suppose that $k$ is positive. Then $M$ is analytically isometric with (i) a complex projective space with a Fubini-Study metric (cf. N. S. Hawley [7], J. Igusa [8]), and simply connected.

Next assume that $k$ is nonpositive. If $M$ is not simply connected, then we have a closed geodesic $l$ which is relatively the shortest and represents a nontrivial class of the 1-dimensional homotopy group. Let $L$ be the length of $l$ and $P$ be an arbitrary point of this geodesic. Take a tangent unit vector $Y_{P}$ to $l$ at $P$. Then by holomorphic free mobility, $Y_{P}$ may be transformed to any direction $Y_{P}^{\prime}$ at $P$ by some automorphism which leaves $P$ invariant. This means that any geodesic starting at $P$ is of the same length $L$, and $l$ must have a conjugate point. This is impossible in a space of nonpositive curvature. Therefore $M$ is simply connected. By a result of J. Igusa [8], any simply connected Kaehlerian manifold with nonpositive constant holomorphic sectional curvature $k$ admits an analytic isometry $\phi$ of $M$ into (ii) a unitary space $(k=0)$, or (iii) an open ball with a Kaehlerian structure of negative constant holomorphic sectional curvature. However, since our manifold is complete, we can conclude that $\phi$ is onto (also cf. [13]). Any of these spaces (i), (ii) and (iii) admits $A(M)$ of the maximum dimension. This completes the proofs of Theorem 3.3 and Theorem A.

REMARK 3.4. If an almost Hermitian manifold admits holomorphic free mobility, then it is a simply connected, homogeneous Kaehlerian manifold with constant holomorphic sectional curvature. However, even if a simply connected homogeneous almost Hermitian manifold $M$ has constant holomorphic sectional curvature, it does not have (local) holomorphic free mobility, as is seen in the 6-dimensional sphere (cf. A. Frölicher [5], T. Fukami and S. Ishihara [6]).

There are many results on the conditions for certain vector fields (affine, conformal, etc.) to be infinitesimal automorphisms. If we take up a theorem of $\mathrm{S}$. Kobayashi and K. Nomizu [10] for example, then it says that in a complete nondegenerate Kaehlerian manifold any infinitesimal affine transformation is an infinitesimal automorphism. So we have

COROllaRY 3.5. Let $M$ be a $2 n$-dimensional complete nondegenerate Kaehlerian manifold. If the dimension of the group of affine transformations is $n(n+2)$, then $M$ is simply connected and has constant holomorphic sectional curvature.

REMARK 3.6. If the unitary group $U(n)$ does not contain any proper subgroup of $r$-dimension such that $n^{2}>r>s$ for some integer $s$, then the condition $\operatorname{dim} A(M)=$ $n(n+2)$ in Theorem 3.3 may be replaced by $\operatorname{dim} A(M)>s+2 n$ and homogeneity.

4. The group of $J$-preserving transformations. Generally, the group $J(M)$ of all $J$-preserving transformations on an almost complex manifold $M$ is infinite dimensional. If $M$ is a bounded domain $D^{n}$ in the complex number space $C^{n}$, then $J(M)$ is a finite dimensional Lie group (H. Cartan [3]). In fact, $D^{n}$ admits the so-called Bergmann metric $G$ which is invariant by holomorphic transformations. Thus $J(M)$ is a subgroup of the group of isometries with respect to $G$ (cf. S. Kobayashi 
[9]). And the maximum dimension of $J(M)$ is $n(n+2)$, which is attained only when $M$ is a simply connected, homogeneous Kaehlerian manifold with negative constant holomorphic sectional curvature.

Also, if $M$ is compact, then $J(M)$ is a finite dimensional Lie group, as was proved first by W. M. Boothby, S. Kobayashi and H. C. Wang [2]. In this section under rather strong conditions on manifolds, we give the maximum dimension of $J(M)$.

Lemma 4.1 (S. TACHIBANA [16]). Let $M$ be a compact almost Kaehlerian manifold. Then we have:

(i) Any Killing vector field is J-preserving.

(ii) If the Ricci form is negative definite, then there is no infinitesimal J-preserving transformation.

(iii) If the Ricci form is negative semidefinite, then any infinitesimal J-preserving transformation is a parallel vector field.

A result due to Y. Matsushima [14] was generalized to two directions by A. Lichnerowicz and S. Sawaki.

LEMMA 4.2 (A. LiCHNEROWICZ [12]). Any infinitesimal J-preserving transformation $X$ on a compact Kaehlerian manifold with the constant scalar curvature $S$ is decomposed into

$$
X=Y+J Z
$$

for some Killing vector field $Y$ and $Z$ (not always unique).

Lemma 4.3 (S. SawaKi [15]). Any infinitesimal J-preserving transformation $X$ on a compact Einstein $(S=$ constant $>0)$ almost Kaehlerian manifold is uniquely decomposed into (4.1) for some Killing vector fields $Y$ and $Z$.

Now we prove one part of Theorem B.

THEOREM 4.4. Let $M$ be a 2n-dimensional compact Einstein almost Kaehlerian manifold with the constant scalar curvature $S$. Then the maximum dimension of $J(M)$ is $2 n(n+2)$. The maximum is attained, if and only if $M$ is analytically isometric with a complex projective space.

Proof. If the scalar curvature $S$ is negative or vanishing, then the Ricci form is negative definite or semidefinite. Hence the dimension of $J(M)$ is at most $2 n$ by Lemma 4.1 (ii) and (iii). Next assume that $S$ is a positive constant. By Lemma 4.3 any infinitesimal $J$-preserving transformation $X$ is of the form $Y+J Z$ for some Killing vector fields $Y$ and $Z$. On the other hand, any Killing vector field is an infinitesimal automorphism by Lemma 4.1 (i). Thus the dimension of $J(M)$ is at most twice $\operatorname{dim} A(M)$, that is, $2 n(n+2)$. If the maximum dimension is attained, then we have $\operatorname{dim} A(M)=n(n+2)$. Since $M$ is compact, by Theorem $\mathrm{A}, M$ is analytically isometric with a complex projective space. The converse is clear. 
THeOREM 4.5. Let $M$ be a 2n-dimensional compact Kaehlerian manifold with the constant scalar curvature $S$. Then the maximum dimension of $J(M)$ is $2 n(n+2)$. The maximum is attained if and only if $M$ is analytically isometric with a complex projective space.

Proof. By Lemma 4.2 we have a decomposition (4.1) of any infinitesimal $J$-preserving transformation $X$. Although this decomposition is not unique in general, the maximum dimension of $J(M)$ is $2 n(n+2)$. Suppose that the maximum is attained. Then the decomposition (4.1) is unique and we have $\operatorname{dim} A(M)=$ $n(n+2)$. This completes the proof.

5. The group of isometries. We denote by $I(M)$ the group of isometries of a $2 n$-dimensional almost Hermitian manifold $M$. The maximum dimension of $I(M)$ is $2 n(2 n+1) / 2=n(2 n+1)$, and is attained in almost Hermitian manifolds of constant curvature, for example, the 6-dimensional sphere [6]. However, as we see by Lemma 4.1 (i) and Theorem A, we have

COROllary 5.1. Let $M$ be a compact $2 n$-dimensional almost Kaehlerian manifold. Then the maximum dimension of $I(M)$ is $n(n+2)$. The maximum is attained if and only if $M$ is a complex projective space.

REMARK 5.2. In the above corollary, we can replace $I(M)$ by the group of conformal transformations if $n>1$, since any infinitesimal conformal transformation on such a space is an infinitesimal automorphism (cf. [16]). Also we can replace $I(M)$ by the group of affine transformations.

\section{REFERENCES}

1. S. Bochner, Curvature in Hermitian metric, Bull. Amer. Math. Soc. 52 (1947), 177-195.

2. W. M. Boothby, S. Kobayashi and H. C. Wang, A note on mappings and automorphisms of almost complex manifolds, Ann. of Math. 77 (1963), 329-334.

3. H. Cartan, Sur les groupes de transformations analytiques, Actualités Sci. Indust., No. 198, Hermann, Paris, 1935.

4. H. Chu and S. Kobayashi, The automorphism group of a geometric structure, Trans. Amer. Math. Soc. 113 (1964), 141-150.

5. A. Frölicher, Zur Differentialgeometrie der komplexen Strukturen, Math. Ann. 129 (1955), 50-95.

6. T. Fukami and S. Ishihara, Almost Hermitian structure on $S^{6}$, Tôhoku Math. J. 7 (1955), 151-156.

7. N. S. Hawley, Constant holomorphic curvature, Canad. J. Math. 5 (1953), 53-56.

8. J. Igusa, On the structure of a certain class of Kaehler varieties, Amer. J. Math. 76 (1954), 669-678.

9. S. Kobayashi, Geometry of bounded domains, Trans. Amer. Math. Soc. 92 (1959), 267-290.

10. S. Kobayashi and K. Nomizu, On automorphisms of a Kaehlerian structure, Nagoya Math. J. 11 (1957), 115-124.

11. - Foundations of differential geometry, Vol. I, Interscience, New York, 1963.

12. A. Lichnerowicz, Géométrie des groupes de transformations, Dunod, Paris, 1958. 
13. Lu Qu-Keng, On the complete Kaehler manifolds with constant unitary curvature, Acta Math. Sinica 16 (1966), 269-281.

14. Y. Matsushima, Sur la structure du groupes d'homéomorphismes analytiques d'une certaine variété kaehlérienne, Nagoya Math. J. 11 (1957), 145-150.

15. S. Sawaki, A generalization of Matsushima's theorem, Math. Ann. 146 (1962), 279-286.

16. S. Tachibana, On almost analytic vectors in almost Kaehlerian manifolds, Tôhoku Math. J. 11 (1959), 247-265.

17. K. Yano and I. Mogi, On real representations of Kaehlerian manifolds, Ann. of Math. 61 (1955), 170-189.

\author{
TÔHOKU UNIVERSITY, \\ SENDAI, JAPAN \\ UNIVERSITY OF ILLINOIS, \\ URBANA, ILLINOIS
}

